Mathematical Research Letters 5, 583-598 (1998)

\title{
A COMBINATORIAL FORMULATION FOR THE SEIBERG-WITTEN INVARIANTS OF 3-MANIFOLDS
}

\author{
Vladimir TURAeV
}

\section{Introduction}

The Seiberg-Witten invariant of a closed connected oriented 3-manifold $M$ is an integer-valued function $S W=S W(M)$ on the set of $\operatorname{Spin}^{c}$-structures $\mathcal{S}(M)$ on $M$. This function is defined under the assumption $b_{1}(M) \geq 1$ where $b_{1}$ is the first Betti number; in the case $b_{1}(M)=1$ the function $S W$ depends on the choice of a generator of the group $H^{1}(M ; \mathbb{Z})=\mathbb{Z}$. The definition of $S W$ runs parallel to the definition of the Seiberg-Witten invariant of 4-manifolds, cf. [Mo], [MT], [MOY].

It was observed by Meng and Taubes [MT] that a weaker function $\underline{S W}(M)$ is essentially equivalent to the Alexander polynomial of $M$. Their proof of this remarkable theorem is based on the interpretation of the Alexander polynomial as a Reidemeister-type torsion, see [Mi] for the case of 3-manifolds with boundary and [Tu1] for the case of closed 3-manifolds.

In 1989, the author introduced so-called Euler structures on manifolds and their combinatorial torsion invariants, see [Tu4]. In dimension 3, the Euler structures are equivalent to $\operatorname{Spin}^{c}$-structures. Combining these facts with the constructions of torsions introduced in the author's earlier papers, one obtains a combinatorially defined function $T=T(M): \mathcal{S}(M) \rightarrow \mathbb{Z}$, see [Tu5]. This function is well defined for $b_{1}(M) \geq 2$ and depends on the choice of a generator of $H^{1}(M ; \mathbb{Z})=\mathbb{Z}$ for $b_{1}(M)=1$. The function $T$ determines the Alexander polynomial of $M$. These facts and the Meng-Taubes theorem strongly suggest a close relationship between the functions $S W$ and $T$.

The following theorem is the main result of this paper.

Theorem 1. For any closed connected oriented 3-manifold $M$ with $b_{1}(M) \geq 1$, we have $S W(M)= \pm T(M)$.

This theorem implies that for any $\operatorname{Spin}^{c}$-structure $s$ on $M$ we have $S W(s)=$ $\pm T(s)$ where the sign \pm is determined by $M$ and does not depend on $s$. This yields a combinatorial computation of the Seiberg-Witten invariant at least up to

Received March 21, 1998. 
sign. A more general theorem including the case of 3-manifolds with boundary will be formulated in Section 4 .

There are reasons to believe that the sign \pm in Theorem 1 is always + so that $S W=T$. A proof of this would require a careful treatment of signs and orientations in the Seiberg-Witten theory (for more on this, see Section 4.4).

The definition of the Seiberg-Witten invariants applies also to 3-dimensional rational homology spheres and yields a metric-dependent function $\mathcal{S} \rightarrow \mathbb{Z}$. For a study of the resulting parameter-dependent invariants, see $[\mathrm{OT}]$. P. Kronheimer introduced a metric-independent version of SW for rational homology spheres by adding a compensation term. Y. Lim and W. Chen proved that this version is equivalent to the Casson invariant. The torsion function $T$ is well-defined for 3 -dimensional rational homology spheres and takes values in $\mathbb{Q}$. It would be interesting to find an analytical interpretation of $T$ in this case.

Our proof of Theorem 1 is indirect. We formulate certain axioms for an abstract numerical invariant of $\operatorname{Spin}^{c}$-structures on 3-manifolds and show that there is at most one invariant (up to sign) satisfying these axioms. It turns out that both $S W$ and $T$ satisfy the axioms, hence $S W= \pm T$. One of the main axioms is a gluing formula standard in the theory of torsions and established by Meng and Taubes $[\mathrm{MT}]$ for the SW-invariants via hard analytical computations. The proof of Theorem 1 is similar in spirit to the Meng-Taubes argument in $[\mathrm{MT}]$, the essential difference is that they knew only a weaker version of $T$ corresponding to the Alexander polynomial (= Milnor's torsion). Note that they used an axiomatic characterization of the multivariable Alexander polynomial of links in $S^{3}$ due to the author, cf. [Tu3]. It would be most interesting to give a direct proof of the equality $S W= \pm T$. For a possible approach, see [HL1], [HL2].

The paper consists of four sections. In Section 1 we recall basic facts concerning the Spin ${ }^{c}$-structures on 3-manifolds and introduce so-called relative Spin $^{c}$-structures. In Section 2 we formulate our axioms for a numerical invariant of $S_{\text {pin }}{ }^{c}$-structures. In Section 3 we show that there is at most one invariant satisfying these axioms. In Section 4 we briefly argue that both $S W$ and $T$ satisfy the axioms and deduce that $S W= \pm T$.

Notation. Throughout the paper the homology and cohomology are taken with integer coefficients unless explicitly indicated to the contrary.

\section{Spin $^{c}$-structures on 3-manifolds}

1.1. $\operatorname{Spin}^{c}$-structures on closed 3-manifolds. Let $M$ be a closed oriented 3-manifold. Endow $M$ with a Riemannian metric and consider the associated principal $S O(3)$-bundle of oriented, orthonormal frames $f_{M}: \operatorname{Fr}(M) \rightarrow M$. Recall that $S O(3)=S U(2) /\{ \pm 1\}=U(2) / U(1)$ where $U(1)$ is the center $(=$ the diagonal subgroup) of $U(2)$. A $S p i n^{c}$-structure on $M$ is a lift of $f_{M}$ to a principal 
$U(2)$-bundle. More precisely, a $\operatorname{Spin}^{c}$-structure on $M$ is an isomorphism class of a pair (a principal $U(2)$-bundle $F \rightarrow M$, an isomorphism of the principal $S O(3)$-bundle $F / U(1) \rightarrow M$ onto $\left.f_{M}: F r(M) \rightarrow M\right)$. The set of Spin ${ }^{c_{-}}$ structures on $M$ is denoted by $\mathcal{S}(M)$.

The group $H_{1}(M)=H^{2}(M)$ acts on $\mathcal{S}(M)$ as follows. If $E \rightarrow M$ is a principal $U(1)$-bundle corresponding to an element of $H^{2}(M)$ and if $F \rightarrow M$ is a $\operatorname{Spin}^{c}$-structure on $M$, then $U(1)$ acts on $E \times F$ in the diagonal way and we obtain a principal $U(2)$-bundle $(E \times F) / U(1) \rightarrow M$. Analyzing the fibre bundle $B U(2) \rightarrow B S O(3)$ induced by the projection $U(2) \rightarrow S O(3)$ it is easy to observe that the action of $H_{1}(M)=H^{2}(M)$ on $\mathcal{S}(M)$ is free and transitive. The action of $H_{1}(M)$ on $\mathcal{S}(M)$ and the group operation in $H_{1}(M)$ will be written multiplicatively.

Using the determinant representation det $: U(2) \rightarrow U(1)$, every Spin $^{c_{-}}$ structure $s \in \mathcal{S}(M)$ defines an associated complex line bundle, $\operatorname{det}(s)$. Its first Chern class defines a mapping $\mathcal{S}(M) \rightarrow H^{2}(M)=H_{1}(M)$ denoted $c$. It follows from definitions that $c(h s)=h^{2} c(s)$ for any $s \in \mathcal{S}(M)$ and $h \in H_{1}(M)$.

1.2. Relative $S p i n^{c}$-structures. The notion of a $S p i n^{c}$-structure readily extends to 3-manifolds with boundary. We shall consider here only 3-manifolds whose boundary consists of tori and define relative Spin $^{c}$-structures extending the canonical Spin $^{c}$-structure on the boundary.

Let $M$ be a compact oriented 3-manifold whose boundary consists of tori. Let us endow $M$ with a Riemannian metric. The restriction of the tangent bundle $T M$ to $\partial M$ splits as a direct sum $\mathbb{R} \oplus T(\partial M)$ where $\mathbb{R}$ is the trivial line bundle over $\partial M$. (We agree that the tangent vector looking outward $M$ corresponds to $+1 \in \mathbb{R})$. The orientation of $M$ induces an orientation of $\partial M$, so that $T(\partial M)$ is an $S O(2)$-bundle. This bundle admits a canonical trivialisation (or rather a canonical homotopy class of trivialisations). On each component $X$ of $\partial M$ the trivialisation is induced by a decomposition of $X$ as the product of two oriented circles. Considered up to homotopy, this trivialisation of $T X$ does not depend on the choice of decomposition $X=S^{1} \times S^{1}$. Indeed, it is invariant under the Dehn twists along the circles $S^{1} \times p t$ and $p t \times S^{1}$. Thus, the bundle $\left.T M\right|_{\partial M}$ has a canonical trivialisation which is well defined up to homotopy fixing the first vector. Therefore $\left.T M\right|_{\partial M}$ admits a canonical $S_{p i n}{ }^{c}$-structure.

Consider the principal $S O(3)$-bundle of oriented orthonormal frames, $f_{M}$ : $\operatorname{Fr}(M) \rightarrow M$. By a relative Spin ${ }^{c}$-structure on $M$, we mean a lift of $f_{M}$ to a principal $U(2)$-bundle whose restriction to $\partial M$ is induced by the canonical trivialisation of $\left.T M\right|_{\partial M}$. More precisely, a relative Spin $^{c}$-structure on $M$ is an isomorphism class of a triple (a principal $U(2)$-bundle $F \rightarrow M$, an isomorphism $\alpha$ of the principal $S O(3)$-bundle $F / U(1) \rightarrow M$ onto $f_{M}: F r(M) \rightarrow M$, a homotopy class of sections $\beta$ of the principal $U(2)$-bundle $\left.F\right|_{\partial M} \rightarrow \partial M$ inducing the 
canonical trivialisation of $\left.\left.T M\right|_{\partial M}\right)$. The condition on $\beta$ means that projecting $\beta$ to $F / U(1)$ and applying $\alpha$ we obtain the canonical trivialisation of $\left.T M\right|_{\partial M}$. By homotopy of $\beta$ we mean a homotopy in the class of sections satisfying this condition.

The set of relative $S p i n^{c}$-structures on $M$ is denoted by $\mathcal{S}(M)$. Representing elements of $H^{2}(M, \partial M)$ by principal $U(1)$-bundles over $M$ trivialised over $\partial M$ and using the construction described in Section 1.1, we obtain an action of $H_{1}(M)=H^{2}(M, \partial M)$ on $\mathcal{S}(M)$. This action is free and transitive so that $\mathcal{S}(M)$ is a principal homogeneous set over $H_{1}(M)$.

Using the determinant det $: U(2) \rightarrow U(1)$, every $s \in \mathcal{S}(M)$ determines an associated complex line bundle, trivialised over $\partial M$. Its first relative Chern class belongs to $H^{2}(M, \partial M)=H_{1}(M)$. This defines a mapping $c: \mathcal{S}(M) \rightarrow H_{1}(M)$. We have $c(h s)=h^{2} c(s)$ for any $s \in \mathcal{S}(M), h \in H_{1}(M)$.

The notion of a $\operatorname{Spin}^{c}$-structure on $M$ is essentially independent of the choice of a Riemannian metric on $M$.

Example 1.3. Let $M$ be the oriented solid torus $S^{1} \times D^{2}$ where $D^{2}$ is a 2-disc. Orient $S^{1}$ and denote by $t$ the generator of $H_{1}(M)$ represented by $S^{1}$. Fix a relative $\operatorname{Spin}^{c}$-structure $s$ on $M$. Then any relative $\operatorname{Spin}^{c}$-structure on $M$ can be uniquely presented as $t^{n} s$ with $n \in \mathbb{Z}$. The formula $c\left(t^{n} s\right)=t^{2 n} c(s)$ implies that the mapping $c: \mathcal{S}(M) \rightarrow H_{1}(M)$ is injective. It is easy to see that its image consists of all odd powers of $t$ (recall that we use multiplicative notation for the group operation in $\left.H_{1}\right)$. Indeed, the relative Chern class $c(s) \in H_{1}(M)$ agrees modulo 2 with the relative Stiefel-Whitney class $w_{2} \in H^{2}(M, \partial M ; \mathbb{Z} / 2 \mathbb{Z})=H_{1}(M ; \mathbb{Z} / 2 \mathbb{Z})$ which is the first obstruction to extending the canonical trivialisation of $\left.T M\right|_{\partial M}$ to $M$. It is clear that this obstruction is non-zero and therefore $c(s)$ is an odd power of $t$. In particular, there is a unique $s_{t} \in \mathcal{S}\left(S^{1} \times D^{2}\right)$ such that $c\left(s_{t}\right)=t$.

1.4. Gluing of $\operatorname{Spin}^{c}$-structures. Let $M$ be a compact oriented 3-manifold whose boundary is either void or consists of tori. Assume that we have a finite family of disjoint embedded tori $\Sigma \subset M$ which splits $M$ as a union of two 3dimensional submanifolds $M_{0}, M_{1}$. Here $M_{0} \cup M_{1}=M$ and $M_{0} \cap M_{1}=\Sigma$. The orientation of $M$ induces orientations of $M_{0}$ and $M_{1}$. Clearly, both $\partial M_{0}$ and $\partial M_{1}$ consist of tori.

There is a natural mapping $\mathcal{S}\left(M_{0}\right) \times \mathcal{S}\left(M_{1}\right) \rightarrow \mathcal{S}(M)$ called the gluing of Spin $^{c}$-structures and defined as follows. Let $s_{0} \in \mathcal{S}\left(M_{0}\right)$ and $s_{1} \in \mathcal{S}\left(M_{1}\right)$. We represent $s_{r}$ by a triple (a principal $U(2)$-bundle $F_{r} \rightarrow M_{r}$, an isomorphism $\alpha_{r}$ of the principal $S O(3)$-bundle $F_{r} / U(1) \rightarrow M_{r}$ onto $\operatorname{Fr}\left(M_{r}\right) \rightarrow M_{r}$, a homotopy class of sections $\beta_{r}$ of the principal $U(2)$-bundle $\left.F_{r}\right|_{\partial M_{r}} \rightarrow \partial M_{r}$ inducing the canonical trivialisation of $\left.T M_{r}\right|_{\partial M_{r}}$ ). The bundles $F_{0} \rightarrow M_{0}$ and $F_{1} \rightarrow M_{1}$ are trivial over $\Sigma$ and moreover have distinguished sections $\left.\beta_{0}\right|_{\Sigma}$ and $\left.\beta_{1}\right|_{\Sigma}$ over $\Sigma$. The projection of $\left.\beta_{r}\right|_{\Sigma}$ to $F_{r} / U(1)$ is mapped by $\alpha_{r}$ into a framing $\left(e_{1}^{r}, e_{2}^{r}, e_{3}^{r}\right)$ 
of $\left.T M\right|_{\Sigma}$ where $\left(e_{1}^{r}, e_{2}^{r}\right)$ is a canonical trivialisation of $T \Sigma$ (with orientation of $\Sigma$ induced from $M_{r}$ ) and $e_{3}^{r}$ is the vector looking outward $M_{r}$. We can assume that $e_{1}^{1}=e_{1}^{0}, e_{2}^{1}=-e_{2}^{0}, e_{3}^{1}=-e_{3}^{0}$. Now, we split $M$ as a union of a cylinder neighborhood $C=\Sigma \times[0,1]$ of $\Sigma=\Sigma \times(1 / 2)$ and copies of $M_{0}$ and $M_{1}$. The notation is chosen so that $C \cap M_{r}=\Sigma \times r$ for $r=0,1$. We glue a principal $U(2)$-bundle $F \rightarrow M$ from three pieces: the bundles $F_{r} \rightarrow M_{r}$ with $r=0,1$ and the trivial bundle $C \times U(2) \rightarrow C$. The gluing identifies $\left.\beta_{r}\right|_{\Sigma}$ with the constant section $x \mapsto x \times 1$ over $\Sigma \times r \subset \partial C$, for $r=0,1$. The bundle isomorphism $F / U(1)=F r(M)$ is also glued from three pieces: the isomorphisms $\alpha_{r}: F_{r} / U(1) \rightarrow \operatorname{Fr}\left(M_{r}\right)$ for $r=0,1$ and the isomorphism $C \times S O(3) \rightarrow \operatorname{Fr}(C)$ which sends a point $(x, t, A)$ with $x \in \Sigma, t \in[0,1], A \in S O(3)$ into the framing $\left(e_{1}^{0}, \cos (\pi t) e_{2}^{0}+\sin (\pi t) e_{3}^{0}, \sin (\pi t) e_{2}^{0}+\cos (\pi t) e_{3}^{0}\right) A$ in the tangent vector space at the point $(x, t) \in C$. This yields a well defined pairing $\mathcal{S}\left(M_{0}\right) \times \mathcal{S}\left(M_{1}\right) \rightarrow \mathcal{S}(M)$ denoted $\cup$. This pairing is bilinear: for any $s_{r} \in \mathcal{S}\left(M_{r}\right), h_{r} \in H_{1}\left(M_{r}\right)$ with $r=0,1$,

$$
\left(h_{0} s_{0}\right) \cup\left(h_{1} s_{1}\right)=i_{0}\left(h_{0}\right) i_{1}\left(h_{1}\right)\left(s_{0} \cup s_{1}\right),
$$

where $i_{r}$ is the inclusion homomorphism $H_{1}\left(M_{r}\right) \rightarrow H_{1}(M)$.

\section{Axioms for an invariant of $\operatorname{Spin}^{c}$-structures}

2.1. The basic setting. A 3-manifold $M$ is said to be homology oriented if the vector space $H_{*}(M ; \mathbb{R})=\oplus_{i=0}^{3} H_{i}(M ; \mathbb{R})$ is oriented. For closed $M$ with $b_{1}(M)=1$, we shall need to specify a generator of the infinite cyclic group $H_{1}(M) /$ Tors $H_{1}(M)$. We say that $M$ is $H_{1}$-directed if either $\partial M \neq \emptyset$, or $b_{1}(M) \neq$ 1 , or $\partial M=\emptyset, b_{1}(M)=1$, and the group $H_{1}(M) /$ Tors $H_{1}(M)$ is endowed with a distinguished generator.

Denote by $\mathcal{M}$ the class of compact, connected, oriented, homology oriented, and $H_{1}$-directed 3 -manifolds whose boundary is either void or consists of tori and whose first Betti number $b_{1}$ is non-zero. Note that the latter condition is superfluous in the case of non-void boundary because $b_{1}(M) \geq b_{1}(\partial M) / 2$ for any compact oriented 3-manifold $M$.

Denote by $\mathcal{S}$ the class of pairs (a 3-manifold $M \in \mathcal{M}$, a relative Spin $^{c}$ structure $s$ on $M$ ). Throughout Section 2 we asume that we have a mapping $v: \mathcal{S} \rightarrow \mathbb{Z}$. We shall formulate four axioms on $v$. Here is the first axiom.

Axiom 1 (Topological invariance). If two pairs $(M, s),\left(M^{\prime}, s^{\prime}\right) \in \mathcal{S}$ are homeomorphic, i.e., if there is a homeomorphism $M \rightarrow M^{\prime}$ preserving the orientation, the homology orientation, the distinguished generator of $H_{1} /$ Tors $H_{1}$ in the case $b_{1}=1, \partial=\emptyset$ and transforming $s$ into $s^{\prime}$, then $v(M, s)=v\left(M^{\prime}, s^{\prime}\right)$.

The remaining three axioms will be formulated in Sections 2.2, 2.3 and 2.4, respectively. 
2.2. Support of $v$. It is convenient to split the second axiom in two parts concerning the cases $b_{1} \geq 2$ and $b_{1}=1$, respectively.

Axiom 2 (first part). For any 3-manifold $M \in \mathcal{M}$ with $b_{1}(M) \geq 2$, the set $\{s \in \mathcal{S}(M) \mid v(M, s) \neq 0\}$ is finite.

Axiom 2 suggests the following notation. For a 3-manifold $M \in \mathcal{M}$, denote by $\mathbb{Z}[[\mathcal{S}(M)]]$ the additive group of functions $\mathcal{S}(M) \rightarrow \mathbb{Z}$. We shall identify a function $f: \mathcal{S}(M) \rightarrow \mathbb{Z}$ with the formal sum $\sum_{s \in \mathcal{S}(M)} f(s) s$. In this way, the additive group of functions with finite support is identified with the additive group $\mathbb{Z}[\mathcal{S}(M)]$ consisiting of finite formal linear combinations of elements of $\mathcal{S}(M)$ with integer coefficients.

Denote by $v(M)$ the function $s \mapsto v(M, s): \mathcal{S}(M) \rightarrow \mathbb{Z}$. We write formally

$$
v(M)=\sum_{s \in \mathcal{S}(M)} v(M, s) s \in \mathbb{Z}[[\mathcal{S}(M)]]
$$

By Axiom 2, in the case $b_{1}(M) \geq 2$ this sum is finite so that $v(M) \in \mathbb{Z}[\mathcal{S}(M)]$.

For a 3-manifold $M \in \mathcal{M}$ with $b_{1}(M)=1$, the infinite cyclic group $H_{1}(M) /$ Tors has a distinguished generator: in the case $\partial M=\emptyset$ it is provided by the assumption that $M$ is $H_{1}$-directed; in the case $\partial M \neq \emptyset$ we distinguish a generator $t \in H_{1}(M) /$ Tors such that the pair $\left([p t] \in H_{0}(M), t\right)$ defines the given orientation of $H_{*}(M ; \mathbb{R})=\mathbb{R}[p t] \oplus \mathbb{R} t$. Observe that for any $C \in H_{1}(M)$ there is a unique integer $k(C)=k_{t}(C)$ such that $C \in t^{k(C)}$ Tors $H_{1}(M)$. For $s \in \mathcal{S}(M)$, we write $s<0$ if $k(c(s))<0$. We say that a function $f: \mathcal{S}(M) \rightarrow \mathbb{Z}$ has an essentially positive support if the set $\{s \in \mathcal{S}(M) \mid s<0, f(s) \neq 0\}$ is finite.

Axiom 2 (second part). For any 3-manifold $M \in \mathcal{M}$ with $b_{1}(M)=1$, the function $v(M): \mathcal{S}(M) \rightarrow \mathbb{Z}$ has an essentially positive support.

This axiom implies that there is an integer $r$ such that $v(M, s)=0$ for any $s \in \mathcal{S}(M)$ with $k(c(s)) \leq r$.

2.3. Excision formula. Consider a 3-manifold $M \in \mathcal{M}$ and an oriented link $L=L_{1} \cup \ldots \cup L_{m} \subset \operatorname{Int} M$. Let $U_{1}, \ldots, U_{m}$ be disjoint closed regular neighborhoods of $L_{1}, \ldots, L_{m}$ in $\operatorname{Int} M$ and let $E=M \backslash\left(\cup_{i} \operatorname{Int} U_{i}\right)$. Clearly, $E$ is a compact connected 3-manifold with boundary consisting of $\partial M$ and $m$ tori. It is called the exterior of $L$. The orientation of $M$ induces an orientation of $E$ in the obvious way. We shall formulate an axiom relating $v(M)$ and $v(E)$.

To consider $v(E)$, we need to provide $E$ with a homology orientation. By assumption, the vector space $H_{*}(M ; \mathbb{R})$ is oriented. The vector space $H_{*}(M, E ; \mathbb{R})$ $=H_{2}(M, E ; \mathbb{R}) \oplus H_{3}(M, E ; \mathbb{R})$ has a basis represented by the meridianal disks of the solid tori $U_{1}, \ldots, U_{m}$ and the fundamental classes of $U_{1}, \ldots, U_{m}$. (The orientations of the meridianal disks and of $U_{1}, \ldots, U_{m}$ are induced by the orientations of 
$L$ and $M)$. This basis of $H_{*}(M, E ; \mathbb{R})$ yields an orientation of this vector space independent of the numeration of the link components $L_{1}, \ldots, L_{m}$. The orientations of $H_{*}(M ; \mathbb{R})$ and $H_{*}(M, E ; \mathbb{R})$ determine an orientation of $H_{*}(E ; \mathbb{R})$ such that the torsion of the exact homology sequence of the pair $(M, E)$ is positive. Thus, $E$ is homology oriented.

By Example 1.3, there is a unique $s_{i} \in \mathcal{S}\left(U_{i}\right)$ such that $c\left(s_{i}\right) \in H_{1}\left(U_{i}\right)$ is the generator represented by $L_{i}$. The formula $s \mapsto s \cup s_{1} \cup \ldots \cup s_{m}$ defines a mapping $\mathcal{S}(E) \rightarrow \mathcal{S}(M)$. This mapping extends by linearity to a mapping $\mathbb{Z}[\mathcal{S}(E)] \rightarrow \mathbb{Z}[\mathcal{S}(M)]$ denoted by in $_{*}$.

Axiom 3 (Excision formula). If, under the conditions above, $b_{1}(E) \geq 2$ and the homology classes $\left[L_{1}\right], \ldots\left[L_{m}\right] \in H_{1}(M)$ have infinite order in $H_{1}(M)$, then for a certain $\varepsilon= \pm 1$,

$$
\operatorname{in}_{*}(v(E))=\varepsilon \prod_{i=1}^{m}\left(1-\left[L_{i}\right]\right) v(M)
$$

Formula $(*)$ needs a few comments. By Axiom $2, v(E) \in \mathbb{Z}[\mathcal{S}(E)]$ so that the left-hand side of $(*)$ is a well-defined element of $\mathbb{Z}[\mathcal{S}(M)] \subset \mathbb{Z}[[\mathcal{S}(M)]]$. On the right-hand side of $(*)$ we use the pull-back action of the group ring $\mathbb{Z}\left[H_{1}(M)\right]$ on $\mathbb{Z}[[\mathcal{S}(M)]]$ induced by the action of $H_{1}(M)$ on $\mathcal{S}(M)$. More precisely, if $h \in H_{1}(M)$ and $f$ is a function $\mathcal{S}(M) \rightarrow \mathbb{Z}$ then the function $h f: \mathcal{S}(M) \rightarrow \mathbb{Z}$ is defined by $(h f)(s)=f\left(h^{-1}(s)\right)$ for any $s \in \mathcal{S}(M)$.

The condition $b_{1}(E) \geq 2$ in Axiom 3 is not too restrictive. It is automatically fulfilled if $b_{1}(M) \geq 2$ or $m \geq 2$, because $b_{1}(E) \geq b_{1}(M)$ and $b_{1}(E) \geq m$.

Axiom 3 has important implications concerning the case $b_{1}(M)=1$. Assume first that $\partial M=S^{1} \times S^{1}$. It is clear that any element $h \in H_{1}(M)$ can be realised by an oriented knot in Int $M$. If the order of $h$ in $H_{1}(M)$ is infinite then the first Betti number of the exterior of such a knot equals 2. Formula $(*)$ implies that for any $h \in H_{1}(M)$ of infinite order, $(1-h) v(M) \in \mathbb{Z}[\mathcal{S}(M)]$. Similarly, for a closed 3-manifold $M$ with $b_{1}(M)=1$, the product $(1-g)(1-h) v(M)$ belongs to $\mathbb{Z}[\mathcal{S}(M)]$ for any $g, h \in H_{1}(M)$ of infinite order. Note that $v(M)$ may depend on the $H_{1}$-direction of $M$ but the product $(1-g)(1-h) v(M)$ does not depend on it. This follows from $(*)$.

2.4. Normalisation. The remaining fourth axiom for $v$ involves the Alexander polynomial of links in $S^{3}$. Consider an $m$-component link $L$ in $S^{3}$ with exterior $E$. Set $H=H_{1}(E)$ (this is a free abelian group of rank $m$ ). Recall that the multivariable Alexander polynomial $A_{L}$ of $L$ is an element of the group ring $\mathbb{Z}[H]$ defined up to multiplication by $\pm h$ with $h \in H$. The Alexander polynomial of $L$ is completely determined by the fundamental group of $E$. It is often described as a Laurent polynomial in $m$ variables corresponding to the meridianal generators 
of $H$. For our purposes, it is more convenient to view $A_{L}$ as an element of $\mathbb{Z}[H] / \pm H$.

In the next axiom and in the sequel, the sphere $S^{3}$ and the exteriors of links in $S^{3}$ are endowed with the right-handed orientation.

Axiom 4 (Normalisation). Let $L \subset S^{3}$ be an m-component link with $m \geq$ 2. Let $E$ be the exterior of $L$ (with right-handed orientation). Then for any homology orientation of $E$ and any $s \in \mathcal{S}(E)$, the sum

$$
\sum_{h \in H_{1}(E)} v(E, h s) h \in \mathbb{Z}\left[H_{1}(E)\right]
$$

represents the Alexander polynomial $A_{L}$ of $L$.

Note that inclusion (**) follows from Axiom 2, since $b_{1}(E) \geq m \geq 2$. A small computation shows that if the condition of Axiom 4 holds for one $s \in \mathcal{S}(E)$, then it holds for all $s$.

\section{Uniqueness}

Theorem 3.1. Let $v, v^{\prime}: \mathcal{S} \rightarrow \mathbb{Z}$ be two mappings satisfying Axioms 1-4. Then for any 3-manifold $M \in \mathcal{M}, v(M)= \pm v^{\prime}(M)$.

The proof begins with a few lemmas. We assume everywhere in this section that we have two mappings $v, v^{\prime}: \mathcal{S} \rightarrow \mathbb{Z}$, satisfying Axioms 1-4. By the meridian of an oriented knot in $S^{3}$ we shall mean an oriented meridian whose linking number with the knot equals +1 .

Lemma 3.2. Let $M \in \mathcal{M}$ and $w \in \mathbb{Z}[[\mathcal{S}(M)]]$. Suppose that there is a homology class of infinite order $h \in H_{1}(M)$ such that $(1-h) w=0$. If either $w$ has a finite support or $b_{1}(M)=1$ and $w$ has an essentially positive support, then $w=0$.

This simple lemma is essentially algebraic. The case where $w$ has a finite support follows from the fact that $\mathbb{Z}[\mathcal{S}(M)]$ is a free $\mathbb{Z}\left[H_{1}(M)\right]$-module of rank 1 freely generated by any $s \in \mathcal{S}(M)$. The case $b_{1}(M)=1$ of the lemma is quite straightforward.

Lemma 3.3. Let $M$ be the cylinder $S^{1} \times S^{1} \times[0,1]$ provided with an orientation and a homology orientation. Then $v(M)= \pm v^{\prime}(M)= \pm s_{0}$ for a certain $s_{0} \in$ $\mathcal{S}(M)$.

Proof. The cylinder $M$ is homeomorphic to the exterior $E$ of a Hopf link, $L \subset S^{3}$. Composing if necessary such a homeomorphism with an orientation-reversing involution $M \rightarrow M$ we can assume that the given orientation of $M$ corresponds to the right-handed orientation of $E$. We orient $L$ so that the induced homology orientation of its exterior $E$ corresponds to the given homology orientation of $M$. 
(Note that when the orientation of a component of $L$ is reversed, the induced homology orientation of $E$ is also reversed). By Axiom 1, it suffices to prove that $v(E)= \pm v^{\prime}(E)= \pm s_{0}$ for a certain $s_{0} \in \mathcal{S}(E)$.

The Alexander polynomial of $L$ is equal to 1 . Axiom 4 implies that the function $s \mapsto v(E, s): \mathcal{S}(E) \rightarrow \mathbb{Z}$ takes value 0 on all $s \in \mathcal{S}(E)$ with one exception $s_{0}$ where it takes value \pm 1 . Similarly, $v^{\prime}(E, s)=0$ for all $s \in \mathcal{S}(E)$ with one exception $s_{0}^{\prime}$ such that $v^{\prime}\left(E, s_{0}^{\prime}\right)= \pm 1$. It remains to show that $s_{0}=s_{0}^{\prime}$.

The group of the isotopy classes of orientation-preserving homeomorphisms of the torus $S^{1} \times S^{1}$ can be identified with $S L_{2}(\mathbb{Z})$. This group acts (up to isotopy) on $E$ via the product of its action on the torus and the identity on $[0,1]$. It is clear that this action preserves the orientation and the homology orientation of $E$. By Axiom 1, the induced action on $\mathcal{S}(E)$ preserves both $v$ and $v^{\prime}$. Therefore it fixes both $s_{0}$ and $s_{0}^{\prime}$. It is clear that the mapping $s \mapsto c(s): \mathcal{S}(E) \rightarrow H_{1}(E)$ commutes with the action of $S L_{2}(\mathbb{Z})$ on the sets $\mathcal{S}(E)$ and $H_{1}(E)$. The only element of $H_{1}(E)$ fixed by the action of $S L_{2}(\mathbb{Z})$ is the neutral element 1 . Therefore $c\left(s_{0}\right)=c\left(s_{0}^{\prime}\right)=1$. Now, the formula $c(h s)=h^{2} c(s)$ shows that the mapping $c: \mathcal{S}(E) \rightarrow H_{1}(E)$ is injective. Hence, $s_{0}=s_{0}^{\prime}$ and $v(E)= \pm v^{\prime}(E)$.

Lemma 3.4. Let $M$ be the 3-dimensional solid torus $S^{1} \times D^{2}$ provided with an orientation and a homology orientation. Then $v(M)= \pm v^{\prime}(M) \neq 0$.

Proof. As at the beginning of the previous lemma, we can identify $M$ with the exterior of an oriented trivial knot $K \subset S^{3}$. Let $L$ be the (oriented) meridian of $K$ pushed inside $M$. We can view $L$ as a core circle $S^{1} \times p t \subset M=S^{1} \times D^{2}$ where $p t \in \operatorname{Int} D^{2}$. It is clear that $[L] \in H_{1}(M)$ is a generator of $H_{1}(M)=\mathbb{Z}$.

Let $E$ be the exterior of $L$ in $M$ provided with the orientation induced by the one of $M$ and with the homology orientation induced by the given homology orientation of $M$ and the orientation of $L$, cf. Section 2.3. The manifold $E$ is homeomorphic to $S^{1} \times S^{1} \times[0,1]$. By Lemma 3.3, $v(E)= \pm v^{\prime}(E)$. By Axiom 3 ,

$$
(1-[L]) v(M)= \pm \operatorname{in}_{*}(v(E))= \pm \operatorname{in}_{*}\left(v^{\prime}(E)\right)=\varepsilon(1-[L]) v^{\prime}(M)
$$

where in $_{*}$ is the homomorphism $\mathbb{Z}[\mathcal{S}(E)] \rightarrow \mathbb{Z}[\mathcal{S}(M)]$ defined in Section 2.3 and $\varepsilon= \pm 1$. Thus, $(1-[L])\left(v(M)-\varepsilon v^{\prime}(M)\right)=0$. Axiom 2 implies that both $v(M)$ and $v^{\prime}(M)$ have essentially positive supports. Therefore $v(M)-\varepsilon v^{\prime}(M)$ has an essentially positive support. By Lemma 3.2, $v(M)=\varepsilon v^{\prime}(M)$.

Note that $\operatorname{in}_{*}(v(E))= \pm \operatorname{in}_{*}\left(s_{0}\right) \neq 0$ where $s_{0} \in \mathcal{S}(E)$ is defined in Lemma 3.3. Therefore $v(M) \neq 0$.

Lemma 3.5. Let $E$ be the exterior of a link $L=L_{1} \cup \ldots \cup L_{m}$ in $S^{3}$ such that $L_{1}, \ldots, L_{m}$ are unknots and $l k\left(L_{1}, L_{i}\right) \neq 0$ for $i=2,3, \ldots, m$. Then for any homology orientation of $E, v(E)= \pm v^{\prime}(E) \neq 0$. 
Proof. The proof goes by induction on $m$. The case $m=1$ was considered in Lemma 3.4. Let $m \geq 2$. We assume that our claim holds for links with $<m$ components and prove it for an $m$-component link $L$.

Let us orient $L$ so that the given homology orientation of $E$ is induced by the orientation of $L$ and the canonical homology orientation of $S^{3}$ determined by the basis $[p t] \in H_{0}\left(S^{3}\right),\left[S^{3}\right] \in H_{3}\left(S^{3}\right)$. Set $H=H_{1}(E)$. We claim that there is an element $g \in H$ such that $v(E)= \pm g v^{\prime}(E)$. To see this, fix $s_{0} \in \mathcal{S}(E)$. By Axioms 2 and 4, the sums

$$
\sum_{h \in H} v\left(E, h s_{0}\right) h \quad \text { and } \quad \sum_{h \in H} v^{\prime}\left(E, h s_{0}\right) h
$$

belong to $\mathbb{Z}[H]$ and represent the Alexander polynomial of $L$. Then, for a certain $g \in H$,

$$
\begin{gathered}
\sum_{h \in H} v\left(E, h s_{0}\right) h= \pm g \sum_{h \in H} v^{\prime}\left(E, h s_{0}\right) h \\
= \pm \sum_{h \in H} v^{\prime}\left(E, h s_{0}\right) g h= \pm \sum_{h \in H} v^{\prime}\left(E, g^{-1} h s_{0}\right) h .
\end{gathered}
$$

This implies $v(E)= \pm g v^{\prime}(E)$.

We show now that $v(E) \neq 0$. Denote by $M$ the exterior of $L_{1}$ in $S^{3}$. We can view $E$ as the exterior of $L_{2} \cup \ldots \cup L_{m}$ in $M$. The homology class of $L_{i}$ in $H_{1}(M)=\mathbb{Z}$ is non-trivial since $\operatorname{lk}\left(L_{1}, L_{i}\right) \neq 0$. We provide $M$ with the homology orientation which induces (together with the orientations of $L_{2}, \ldots, L_{m}$ ) the given homology orientation of $E$. By Axioms 2, 3 and Lemma 3.2, the equality $v(E)=0$ would imply $v(M)=0$. This contradicts Lemma 3.4. Thus $v(E) \neq 0$. Therefore there exists a unique $g \in H$ such that $v(E)= \pm g v^{\prime}(E) \in$ $\mathbb{Z}[H]$.

Let us prove that $g=1$. Let $t_{1}, \ldots, t_{m}$ be the generators of $H=H_{1}(E)=\mathbb{Z}^{m}$ represented by the (oriented) meridians of $L_{1}, \ldots, L_{m}$. We have $g=\prod_{j=1}^{m} t_{j}^{n_{j}}$ with integer $n_{1}, \ldots, n_{m}$. Fix $i \in\{2,3, \ldots, m\}$. Denote by $N=N_{i}$ the exterior of the link $L_{1} \cup \ldots \cup L_{i-1} \cup L_{i+1} \cup \ldots \cup L_{m}$ in $S^{3}$. It is clear that $E$ is the exterior of $L_{i}$ in $N$. The homology class of $L_{i}$ in $H_{1}(N)$ is non-trivial because $\operatorname{lk}\left(L_{1}, L_{i}\right) \neq 0$. We provide $N$ with the homology orientation which induces (together with the orientation of $L_{i}$ ) the given homology orientation of $E$. By Axiom 3 and the inductive assumption,

$$
\begin{gathered}
\operatorname{in}_{*}(v(E))= \pm\left(1-\left[L_{i}\right]\right) v(N), \\
\operatorname{in}_{*}\left(v^{\prime}(E)\right)= \pm\left(1-\left[L_{i}\right]\right) v^{\prime}(N)= \pm\left(1-\left[L_{i}\right]\right) v(N),
\end{gathered}
$$

where $\operatorname{in}_{*}$ is the inclusion homomorphism $\mathbb{Z}[\mathcal{S}(E)] \rightarrow \mathbb{Z}[\mathcal{S}(N)]$. Combining these equalities with $v(E)= \pm \prod_{j=1}^{m} t_{j}^{n_{j}} v^{\prime}(E)$ and using Lemma 3.2 and the inductive 
assumption $v(N) \neq 0$ we obtain $\prod_{j \neq i} t_{j}^{n_{j}}=1$. Therefore $n_{j}=0$ for all $j \neq i$. If $m>2$, then applying this to $i=2$ and $i=m$ we obtain $n_{j}=0$ for all $j$. If $m=2$, then $i=2$ and we have only $n_{1}=0$. However, by symmetry between $L_{1}$ and $L_{2}$ in the case $m=2$ we have also $n_{2}=0$. Thus, $n_{j}=0$ for all $j$, so that $g=1$ and $v(E)= \pm v^{\prime}(E)$.

Lemma 3.6. Let $E$ be the exterior of a link $L=L_{1} \cup \ldots \cup L_{m}$ in $S^{3}$ such that $L_{1}, \ldots, L_{m}$ are unknots. Then for any homology orientation of $E, v(E)=$ $\pm v^{\prime}(E)$.

Proof. The case $m=1$ being covered by Lemma 3.4, it suffices to consider the case $m \geq 2$. Let $K$ be an oriented knot in $E$ whose linking numbers with all components of $L$ are non-zero. We can choose $K$ such that it is unknotted in $S^{3}$. Let $E_{K}$ be the exterior of $K \cup L$ in $S^{3}$. By the previous lemma, $v\left(E_{K}\right)=$ $\pm v^{\prime}\left(E_{K}\right)$ for any homology orientation of $E_{K}$. Clearly, $E_{K}$ is the exterior of $K$ in E. By Axiom 3,

$$
(1-[K]) v(E)= \pm \operatorname{in}_{*}\left(v\left(E_{K}\right)\right)= \pm \operatorname{in}_{*}\left(v^{\prime}\left(E_{K}\right)\right)= \pm(1-[K]) v^{\prime}(E)
$$

where $[K] \in H_{1}(E)$ is the homology class of $K$. Since $m \geq 2$, both $v(E)$ and $v^{\prime}(E)$ belong to $\mathbb{Z}[\mathcal{S}(E)]$. By Lemma 3.2, $v(E)= \pm v^{\prime}(E)$.

Lemma 3.7. Let $E$ be the exterior of a link $L$ in $S^{3}$. Then for any homology orientation of $E, v(E)= \pm v^{\prime}(E)$.

Proof. Let us call a link in $S^{3}$ weakly trivial if all its components are unknotted. It is well known that there is a link $K \subset S^{3} \backslash L$ such that the exterior, $\tilde{E}$, of $K \cup L$ is homeomorphic (via an orientation preserving homeomorphism) to the exterior of a weakly trivial link in $S^{3}$. Moreover, one can choose $K$ so that all its components are homologically non-trivial in $S^{3} \backslash L$. The previous lemma and Axiom 1 imply that $v(\tilde{E})= \pm v^{\prime}(\tilde{E})$. Then applying Axiom 3 and Lemma 3.2, we obtain $v(E)= \pm v^{\prime}(E)$.

For completeness, we outline the construction of $K$. Let us present $L$ by a link diagram $X$. Switching certain over-crossings to undercrossings we can transform $X$ into a diagram of a weakly trivial link, $L^{\prime}$. In fact, we have to switch only selfcrossings of components since we need only to undo the individual components of $L$. At each crossing $x$ where we make the switch, consider a small unknotted circle $S_{x}^{1}$ which encircles the two branches of $L$ involved in the crossing. This circle bounds a small disc pierced by $L$ twice. We choose $S_{x}^{1}$ so that $L$ pierces this disc twice in the same direction; this condition makes sense for unoriented $L$ because the two branches of the crossing lie on the same component of $L$. Then $S_{x}^{1}$ represents a non-trivial element of $H_{1}\left(S^{3} \backslash L\right)$. Let $K=\cup_{x} S_{x}^{1}$ be the link formed by these unknotted circles appearing at the crossings $x$ of $X$ where 
we make the switch. Then the link $K \cup L^{\prime}$ is weakly trivial. We shall show that the exteriors of $K \cup L$ and $K \cup L^{\prime}$ are homeomorphic. For every crossing $x$ as above, choose a small regular neighborhood $U_{x} \subset S^{3} \backslash L$ of $S_{x}^{1}$. Let $D^{2} \times[0,1]$ be a small cylinder in $S^{3}$ encircled by the solid torus $U_{x}$; this means that

$$
\left(D^{2} \times[0,1]\right) \cap U_{x}=\left(D^{2} \times[0,1]\right) \cap \partial U_{x}=\partial D^{2} \times[0,1],
$$

is an annulus formed by longitutes of $U_{x}$. We assume that $L$ meets each 2 disc $D^{2} \times t$ transversally in two points. Consider a self-homeomorphism of the cylinder $D^{2} \times[0,1]$ rotating $D^{2} \times t$ to the angle $2 \pi t$ around its center in a certain direction. We take the disjoint union of such homeomorphisms acting in the cylinders encircled by $\left\{U_{x}\right\}_{x}$ and extend it to a self-homeomorphism $\varphi$ of $S^{3} \backslash\left(\cup_{x} \operatorname{Int} U_{x}\right)$ acting as the identity outside these cylinders. It is easy to see that for an appropriate choice of the rotation directions above, $\varphi(L)=L^{\prime}$. The homeomorphism $\varphi$ induces a homeomorphism of the exteriors of $K \cup L$ and $K \cup L^{\prime}$.

3.8. Proof of the equality $v= \pm v^{\prime}$ for closed 3-manifolds. Let $M \in \mathcal{M}$ be a closed, connected, oriented, homology oriented, and $H_{1}$-directed 3 -manifold with $b_{1}(M) \geq 1$. Let us prove that $v(M)= \pm v^{\prime}(M)$. It is well known that $M$ can be obtained by the surgery on a framed link in $S^{3}$. Consider the inverse surgery, i.e., a surgery on $M$ along a framed link $L=L_{1} \cup \ldots \cup L_{m} \subset M$ which produces $S^{3}$. We assume that $m \geq 2$ : if $m=1$ then we just add to $L$ an unknotted circle with framing +1 contained in a small ball disjoint from $L$. Fix an arbitrary orientation of $L$. Since the surgery along $L$ gives $S^{3}$, the homology classes $\left[L_{1}\right], \ldots,\left[L_{m}\right]$ generate $H_{1}(M)$. Since $b_{1}(M) \geq 1$, at least one of these classes, say $\left[L_{1}\right] \in H_{1}(M)$, has infinite order. Replacing $L_{i}, i \geq 2$ with its band sum with $L_{1}$, we keep the result of the surgery along $L$ while replacing $\left[L_{i}\right]$ with $\left[L_{i}\right]+\left[L_{1}\right]$. In this way, we can ensure that the homology classes of all the components of $L$ are of infinite order in $H_{1}(M)$.

Denote by $E$ the exterior of $L$ in $M$. By definition of surgery, the sphere $S^{3}$ is obtained by gluing $m$ solid tori to $E$. This implies that $E$ is homeomorphic to the exterior of an $m$-component link in $S^{3}$ via a homeomorphism transforming the orientation of $E$ induced from $M$ into the right-handed orientation. As in Section 2.3, the orientation of $L$ and the homology orientation of $M$ induce a homology orientation of $E$. By Lemma 3.7 and Axiom 1, $v(E)= \pm v^{\prime}(E)$. Axiom 4 applied to the link $L \subset M$ and the functions $v, v^{\prime}$ gives

$$
\prod_{i=1}^{m}\left(1-\left[L_{i}\right]\right) v(M)= \pm \operatorname{in}_{*}(v(E))= \pm \operatorname{in}_{*}\left(v^{\prime}(E)\right)= \pm \prod_{i=1}^{m}\left(1-\left[L_{i}\right]\right) v^{\prime}(M)
$$

By Lemma 3.2, $v(M)= \pm v^{\prime}(M)$. 
3.9. Proof of the equality $v= \pm v^{\prime}$ for 3-manifolds with boundary. Let $M \in \mathcal{M}$ be a compact, connected, oriented, homology oriented 3-manifold with $b_{1}(M) \geq 1$ whose boundary is non-void and consists of tori. We shall prove that $v(M)= \pm v^{\prime}(M)$. First of all, we present $M$ as the exterior of a link, $L^{\prime}$, in a closed, connected, oriented 3-manifold, $N$ such that the meridians of the components of $L^{\prime}$ represent elements of $H_{1}(M)$ of infinite order. To construct $N$ choose on every component of $\partial M$ a simple closed curve representing an element of infinite order in $H_{1}(M)$ and glue 3 -dimensional solid tori to $M$ so that their meridianal discs are glued to these simple closed curves.

As above, we can obtain $S^{3}$ by surgery on a framed link $L=L_{1} \cup \ldots \cup L_{m}$ in $N$ with $m \geq 2$. We can assume that $L$ does not meet $L^{\prime}$. Note that an isotopy of $L_{i}$ in $N$ crossing $L^{\prime}$ transversally changes the homology class $\left[L_{i}\right] \in H_{1}\left(N \backslash L^{\prime}\right)=$ $H_{1}(M)$ by the homology class of the meridian of a component of $L^{\prime}$. Therefore, deforming if necessary $L$ in $N$, we can ensure that $L \subset M \subset N \backslash L^{\prime}$ and the homology classes of $L_{1}, \ldots, L_{m}$ are of infinite order in $H_{1}(M)$. Now, the exterior, $E$, of the link $L \cup L^{\prime}$ in $N$ is homeomorphic to the exterior of a link in $S^{3}$. By Lemma 3.7 and Axiom 1, $v(E)= \pm v^{\prime}(E)$. On the other hand, $E$ is the exterior of $L$ in $M$ so that we can apply Axiom 4. The same argument as in the case of closed manifolds gives $v(M)= \pm v^{\prime}(M)$.

\section{Generalization and proof of Theorem 1}

4.1. The Seiberg-Witten function $S W$. Let $M$ be a compact, connected, oriented, homology oriented, and $H_{1}$-directed 3 -manifold whose boundary is either void or consists of tori and whose first Betti number $b_{1}$ is non-zero. The Seiberg-Witten invariant of $S_{p i n}{ }^{c}$-structures on $M$ is defined in [MT, Section 2]. In the case of closed $M$, this gives a function $S W: \mathcal{S}(M) \rightarrow \mathbb{Z}$. In the case of non-void boundary we need more care. Denote by $\mathcal{S}(M)$ the set of pairs $(S, x)$ where: $S$ is a $U(2)$-structure in the tangent bundle of $M$ whose first Chern class $c_{1}(S) \in H^{2}(M)$ restricts to zero on every component of $\partial M$ and $x \in H^{2}(M, \partial M) /$ Tors such that the image of $x$ under the natural homomorphism $H^{2}(M, \partial M) /$ Tors $\rightarrow H^{2}(M) /$ Tors equals $c_{1}(S)$ (mod Tors). The Seiberg-Witten invariant of $M$, as defined in $[\mathrm{MT}]$, is a function $\underline{\mathcal{S}}(M) \rightarrow \mathbb{Z}$. Now, every relative Spin $^{c}$-structure $s$ on $M$ defines a pair $(S(s), x(s)) \in \underline{\mathcal{S}}(M)$. Here $S(s)$ is obtained by forgetting the section $\beta$ over $\partial M$, see Section 1.2. The cohomology class $x(s)$ is the relative first Chern class $c(s) \in H^{2}(M, \partial M)$ considered modulo Tors . Composing the resulting mapping (in fact, embedding) $\mathcal{S}(M) \rightarrow \underline{\mathcal{S}}(M)$ with the Seiberg-Witten invariant $\mathcal{S}(M) \rightarrow \mathbb{Z}$ we obtain the function $S W: \mathcal{S}(M) \rightarrow \mathbb{Z}$.

We claim that the function $S W$ satisfies Axioms 1-4 of Section 2. Axiom 1 is a basic property of the Seiberg-Witten invariants which are defined using a Riemannian metric on $M$ but do not depend on the choice of the metric. Axiom 2 expresses a fundamental and well known property of the SW-invariants. Axiom 3 
appeared in [MT], where it is formulated up to a certain indeterminacy related to Tors $H_{1}$. According to G. Meng [Me], the proof actually gives the precise formula without any indeterminacy. Axiom 4 follows from Theorem 1.1 of [MT].

4.2. The function $T$. Let $M$ be a compact, connected, oriented, homology oriented, and $H_{1}$-directed 3 -manifold whose boundary is either void or consists of tori and whose first Betti number $b_{1}$ is non-zero. Recall the notion of a (smooth) Euler structure on $M$ introduced in [Tu4]. An Euler structure on $M$ is a nonsingular vector field on $M$ directed outward on $\partial M$ and considered up to homotopy and arbitrary modifications in a small ball in $\operatorname{Int} M$. The set of Euler structures on $M$ is denoted by $\operatorname{Eul}(M)$. The group $H_{1}(M)$ acts on Eul $(M)$ freely and transitively. There is a natural $H_{1}(M)$-equivariant bijection $\operatorname{Eul}(M) \rightarrow \mathcal{S}(M)$ defined as follows. A nonsingular vector field $u$ on $M$ splits the tangent vector bundle of $M$ as a direct sum $\mathbb{R} u \oplus u^{\perp}$. The oriented 2dimensional vector bundle $u^{\perp}$ has the structure group $U(1)$. This reduces the structure group of the tangent bundle of $M$ to $U(1)=1 \oplus U(1) \subset U(2)$. For more details, see [Tu4], [Tu5].

In [Tu5] the author used the theory of torsions to define a function Eul $(M) \rightarrow$ $\mathbb{Z}$. Combining it with the bijection $\operatorname{Eul}(M)=\mathcal{S}(M)$ we obtain a function $T: \mathcal{S}(M) \rightarrow \mathbb{Z}$. We claim that the function $T$ satisfies Axioms 1-4 of Section 2. Axiom 1 is a basic property of torsions which are defined using a triangulation of $M$ but do not depend on the choice of this triangulation. Axiom 2 is established in [Tu5]. Axiom 3 follows from the multiplicativity of torsions; its weaker version appeared already in [Tu2]. Axiom 4 expresses the fact that the Alexander polynomial of a link can be computed as a torsion, see [Mi].

Theorem 4.3 (Generalization of Theorem 1). For any 3-manifold $M \in \mathcal{M}$, we have $S W(M)= \pm T(M): \mathcal{S}(M) \rightarrow \mathbb{Z}$.

Proof. The functions $S W$ and $T$ both satisfy Axioms 1-4. By Theorem 3.1, $S W(M)= \pm T(M)$.

\subsection{Remarks.}

1. An interesting problem is to determine the sign in the equality $S W= \pm T$. For link exteriors in $S^{3}$ this sign is always + . This follows from the fact that for the exterior of an oriented link $L \subset S^{3}$ both functions $S W$ and $T$ determine the Conway-normalised Alexander polynomial of $L$. Recall that the Alexander polynomial $A_{L}$ of $L$ admits a sign-determined normalisation due to Conway (see [Tu3] and references therein). The Conway-normalised Alexander polynomial of $L$ is an element of $\mathbb{Z}[H] / H$ where $H$ is the first homology group of the exterior of $L$. Now we can formulate a more precise version of Axiom 4 for a function $v: \mathcal{S} \rightarrow \mathbb{Z}$ as follows. 
Axiom $4^{\prime}$. Let $L \subset S^{3}$ be an oriented $m$-component link with $m \geq 2$. Let $E$ be the exterior of $L$ with right-handed orientation and homology orientation induced by the orientation of $L$ and the canonical homology orientation of $S^{3}$ (cf. Sections 2.3 and 3.5). Then for any $s \in \mathcal{S}(E)$, the sum

$$
\sum_{h \in H_{1}(E)} v(E, h s) h \in \mathbb{Z}\left[H_{1}(E)\right]
$$

represents the Conway-normalised Alexander polynomial of $L$.

Both functions $v=T$ and $v=S W$ satisfy Axiom $4^{\prime}$. For $v=T$, this follows from the results of [Tu3]. For $v=S W$, this follows from Theorem 1.1 of [MT]. These facts and Theorem 4.3 imply that $S W(E)=T(E)$. To compute the sign in the equality $S W(M)= \pm T(M)$ for an arbitrary 3-manifold $M \in \mathcal{M}$, we need to know the sign \pm in the excision formula for the functions $S W$ and $T$. A computation for $T$ is quite easy; the missing step is a computation of the sign in the excision formula for $S W$.

2. Note a few simple properties of the function $T= \pm S W$, cf. [Tu5]. For $M \in \mathcal{M}$, denote by $\bar{M}$ the same manifold with opposite homology orientation. Then $T(\bar{M}, s)=a-T(M, s)$ for any $s \in \mathcal{S}(M)$, where

$$
a=\left\{\begin{array}{l}
0, \text { if } b_{1}(M) \geq 2 \text { or } b_{1}(M)=1 \text { and } \partial M=\emptyset \\
1, \text { if } b_{1}(M)=1 \text { and } \partial M \neq \emptyset .
\end{array}\right.
$$

Another important property of $T$ is the charge conjugation invariance. It is well known for closed 3-manifolds and may be extended to 3-manifolds with boundary: for any $(M, s) \in \mathcal{S}$, we have

$$
T(M, \bar{s})=(-1)^{b} T(M, s),
$$

where $b=b_{0}(\partial M)$ is the number of connected components of $\partial M$ and where $s \mapsto \bar{s}$ is the involution in $\mathcal{S}(M)$ induced by the involution $a \mapsto(\overline{\operatorname{det}(a)} / \operatorname{det}(a)) a$ in $U(2)$.

We can also describe the behavior of the function $T$ for a closed 3-manifold $M \in \mathcal{M}$ with $b_{1}(M)=1$ under the inversion of the distinguished generator $t \in H_{1}(M) /$ Tors $H_{1}(M)=\mathbb{Z}$. Namely, $T\left(M, t^{-1}, s\right)=T(M, t, s)-k_{t}(s) / 2$, for any $s \in \mathcal{S}(M)$, where $k_{t}: \mathcal{S}(M) \rightarrow \mathbb{Z}$ is the function defined in Section 2.2.

\section{Acknowlegements}

This paper was written during the visit of the author to the Hong Kong University of Science and Technology. The author is sincerely grateful to Guowu Meng for the hospitality and stimulating discussions. 


\section{References}

[HL1] M. Hutchings and Y.-J. Lee, Circle-valued Morse theory, Reidemeister torsion, and Seiberg-Witten invariants of 3-manifolds, preprint dg-ga/9612004.

[HL2] M. Hutchings and Y.-J. Lee, Circle-valued Morse theory and Reidemeister torsion, preprint dg-ga/9706012.

[MT] G. Meng and C.H. Taubes, $\underline{S W}=$ Milnor torsion, Math. Res. Lett. 3 (1996), 661674.

[Me] G. Meng, private communication (February, 1998).

[Mi] J. Milnor, A duality theorem for Reidemeister torsion, Ann. of Math. 76 (1962), $137-147$.

[Mo] J.W. Morgan, The Seiberg-Witten equations and applications to the topology of smooth four-manifolds, Mathematical Notes, 44, Princeton University Press, Princeton, N.J., 1996.

[MOY] T. Mrowka, P. Ozsváth, and B. Yu, Seiberg-Witten monopoles on Seifert fibered spaces (1996), MSRI Preprint.

[OT] C. Okonek and A. Teleman, Three dimensional Seiberg-Witten invariants and nonKählerian geometry, preprint (1998).

[Tu1] V. Turaev, The Alexander polynomial of a three-dimensional manifold, Mat. Sb. (N.S.) 97 (1975), 341-359; English transl. in Math. USSR Sb. 26 (1975), 313-329.

[Tu2] V. Turaev, Reidemeister torsion and the Alexander polynomial, Mat. Sb. (N.S.) 101 (1976), 252-270; English transl. in Math. USSR Sb. 30 (1976), 221-237.

[Tu3] V. Turaev, Reidemeister torsion in knot theory, Uspekhi Mat. Nauk 41 (1986), 97147; English transl. in Russian Math. Surveys 41 (1986), 119-182.

[Tu4] V. Turaev, Euler structures, nonsingular vector fields, and Reidemeister-type torsions, Izvestia Ac. Sci. USSR 53 (1989); English transl. in Math USSR Izvestia 34 (1990), 627-662.

[Tu5] V. Turaev, Torsion invariants of Spin ${ }^{c}$-structures on 3-manifolds, Math. Res. Lett. 4 (1997), 679-695.

Institut de Recherche Mathématique Avancée, Université Louis Pasteur C.N.R.S., 7 rue René Descartes, F-67084 Strasbourg, France

E-mail address: turaev@math.u-strasbg.fr 